\title{
LIGHTNING INDUCED VOLTAGES ON CONDUCTORS INTERLINKING THE TOWERS OF A SATELLITE LAUNCH PAD
}

\author{
P. DURAI KANNU and JOY THOMAS M. \\ DEPARTMENT OF HIGH VOLTAGE ENGINEERING \\ INDIAN INSTITUTE OF SCIENCE \\ BANGALORE 560012, INDIA
}

\begin{abstract}
Modern satellite launch pads are provided with lightning protection system to, take care of direct lightning strike to the satellite launch vehicle during launch preparation period. This paper presents the results of the induced voltage on the conductors interlinking the towers of such a protection system due to a nearby lightning strike. The variation in the induced voltages with lightning strike distances as well as ground conductivities are studied and the results presented in the paper. It has been seen that the waveshape of the induced voltage depends on the : riking distance, where as the earth conductivity has negligible effect on the induced voltage. Computational analysis also shows that the magnitude of the induced voltage is reduced by bundling the conductors interlinking the towers.
\end{abstract}

\section{Introduction}

Lightning poses a serious threat to tall objects. A satellite launch vehicle being tall, it is necessary to prevent direct strikes to it by diverting the lightning strike to a close by relatively safe spot [1]. I modern satellite launch pads are provided with lightning protection system to take care of direct lightning strike to the satellite launch vehicle during launch preparation period.

The launch pad protection system under study consists of three tall towers, each $120 \mathrm{~m}$ in height located at the vertices of an equilateral triangle of base $180 \mathrm{~m}$. There are five conductors each of radius $1.2 \mathrm{~cm}$ connecting the tower top. All the five conductors are bundled at regular intervals throught the span. A simplified diagram of the launch pad protection system is shown in figure1. Any lightning strike coming close to the launch pad is attracted to the tall towers or the conductors and gets diverted to the ground there by avoiding a direct strike to the launch vehicle. However a strike coming close to the protection system but much beyond the striking distance may not terminate at the lightning protection system. But it can induce very high voltage on the lightning protection system conductors as well as the communication and lighting cables

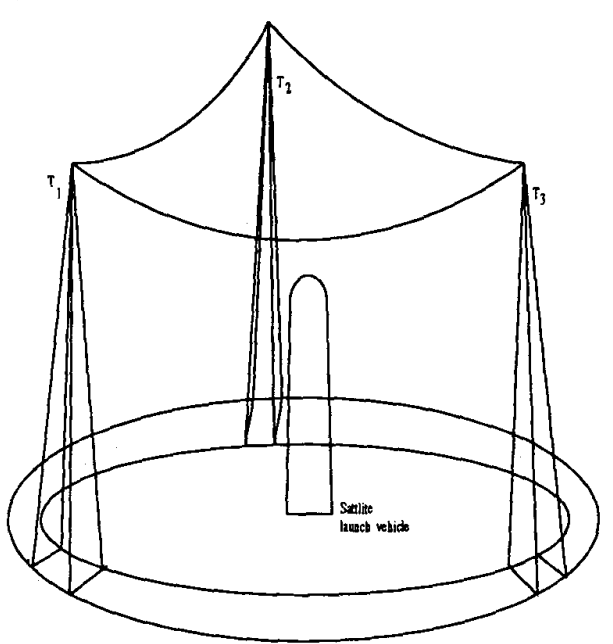

Figure 1: The simplified diagram of launching pad protection system. T's are the three towers.

on the towers by electromagnetic induction. It becomes also necessary to characterise the induced voltage waveforms due to an indirect strike so that the lightning strike monitoring system of the protection scheme can differentiate between a direct strike as well as an indirect strike.

This paper discusses the mathematical model for transient field generated by a nearby lightning stroke as well as'its coupling to the conductors interlinking the towers. Finally induced voltages for various cases have been computed and presented. 
Proceedings of the International Conference on Electromagnetic Interference and Compatibility '99

\section{Methodology for computation of induced voltage}

The evaluation of induced voltage on the overhead conductors due to a near by lightning strike is generally performed in the following way.

1. Developement of a reliable lightning return stroke model. ie., the specification of the spatial and temporal distribution of the lightning current along the channel, which produces the electromagnetic fields similar to those generated by lightning return strokes.

2. Estimation of the distortions introduced in the electromagnetic fields generated by the return stroke as they propagate over finitely conducting ground and there by obtain the vertical and horizontal components of electric field at the overhead conductors.

3. Use a coupling model to simulate the interaction of the electromagnetic fields with the conductors and hence calculate the induced voltage. The conductors between the towers are modeled as transmission line with distributed parameters and electric field produced by the lightning stroke channel is used as the source of excitation.

\section{Lightning return stroke model}

The return-stroke model is an equation for the longitudinal channel current $i\left(z^{\prime}, t\right)$ at height $z^{\prime}$ and for a given time instant $t$. The lightning return stroke model assumes that the current pulse is propagating upward as it would propagate along a uniform transmission line matched at the upper end. The model adopted for this study is the modified transmission line(MTL) model[2]. In this model the lightning current is allowed to decrease with height while propagating along the channel upward. The current propagation along the channel is represented as

$$
i\left(z^{\prime}, t\right)=e^{\left(-z^{\prime} / \lambda\right)} i\left(0, t-z^{\prime} / v\right)
$$

where $v$ is the velocity of the return stroke and $\lambda$ is the decay constant to account for the effect of the vertical distribution of charge stored in the corona sheath of the leader and subsequent discharge during the return stroke phase. The lightning return stroke channel is assumed straight and vertical.

For the lightning stroke current at the ground level ie. $i(0, t)$, double exponential pulse described by the equation (2) is adopted[3].

$$
i(0, t)=i_{0}\left(e^{-\alpha t}-e^{-\beta t}\right)
$$

For the lightning stroke current at the ground level, the values of $\alpha$ and $\beta$ are chosen as $3 \times 10^{8} s^{-1}$ and $10^{7} s^{-1}$ respectively[3] The return stroke velocity $v$ is taken as $1.3 \times 10^{8} \mathrm{~m} / \mathrm{s}$ and decay constant $\lambda$ is taken as $1.7 \mathrm{~km}[2]$.

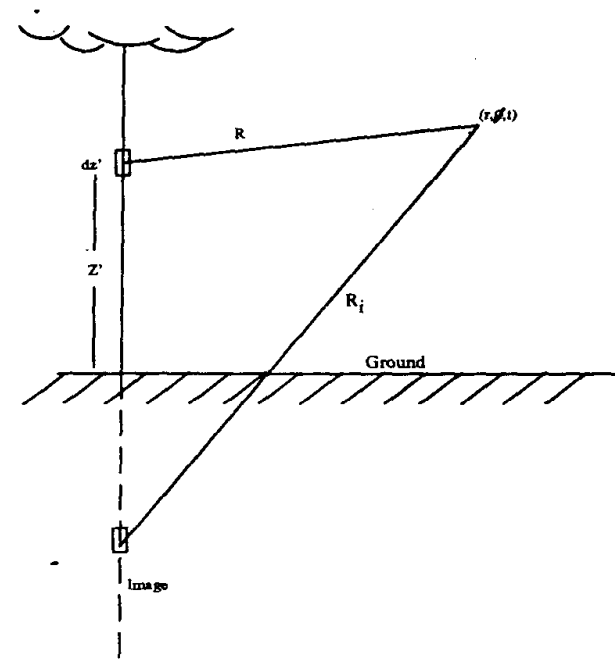

Figure 2: Sketch showing the lightning channel and its image used in calculating the return stroke fields.

\section{Electromagnetic fields generated by lightning}

The determination of the électric and magnetic fields produced $b_{0}:$ the lightning is very similar to that of a vertical antenna radiating above a lossy half-space. The coupling model adopted for the calculation of lightning induced voltage due to lightning return stroke requires, horizontal and vertical components of the electric field produced by the lightning current. The vertical electric field $d E_{z}(r, z, t)$ and the horizontal electric fiel 1 $d E_{r}(r, z, t)$ at altitude $z$ and distance $r$, generated by a vertical dipole of infinitesimal length $d z^{\prime}$ at height $z^{\prime}$ can be calculated by employing equations given below if we assume that the earth is a flat and perfectly conducting surface $[4,5]$.

$$
\begin{aligned}
& \mathrm{dE}_{z}(r, z, t)=\frac{d z^{\prime}}{4 \pi \varepsilon_{0}}[ \\
& \frac{2\left(z-z^{\prime}\right)^{2}-r^{2}}{R^{5}} e^{\left(-z^{\prime} / \lambda\right)} \int_{0}^{t} i\left(0, \tau-z^{\prime} / v-R / c\right) d \tau \\
& +\frac{2\left(z-z^{\prime}\right)^{2}-r^{2}}{c R^{4}} e^{\left(-z^{\prime} / \lambda\right)} i\left(0, t-z^{\prime} / v-R / c\right) \\
& \left.-\frac{r^{2}}{c^{2} R^{3}} e^{\left(-z^{\prime} / \lambda\right)} \frac{\partial}{\partial t} i\left(0, t-z^{\prime} / v-R / c\right)\right] \\
& \\
& \frac{\mathrm{dE}_{r}(r, z, t)=\frac{d z^{\prime}}{4 \pi \epsilon_{0}}[}{R^{5}} e^{\left(-z^{\prime} / \lambda\right)} \int_{0}^{t} i\left(0, \tau-z^{\prime} / v-R / c\right) d \tau \\
& +\frac{3 r\left(z-z^{\prime}\right)}{c R^{4}} e^{\left(-z^{\prime} / \lambda\right)} i\left(0, t-z^{\prime} / v-R / c\right) \\
& \left.+\frac{r\left(z-z^{\prime}\right)}{c^{2} R^{3}} e^{\left(-z^{\prime} / \lambda\right)} \frac{\partial}{\partial t} i\left(0, t-z^{\prime} / v-R / c\right)\right]
\end{aligned}
$$


Kannu \& Thomas : Lightning Induced Voltages on Conductors. Interlinking...

where $\epsilon_{0}$ is the permittivity of free space and $\mathrm{c}$ is the velocity of light. $\mathrm{R}=\sqrt{r^{2}+\left(z-z^{\prime}\right)^{2}}$ is the distance from the dipole to the observation point. The effects of the perfectly conducting ground plane on the electric fields generated by the dipole are included by replacing the ground by an image current dipole at a distance $z^{\prime}$ below the ground reference plane. A sketch showing the lightning channel and its image used in calculating the return stroke fields is shown in figure 2 . The fields due to the image dipole is obtained by substituing $R_{i}$ for $R$ in the equations (3) and (4). The total vertical and horizontal electric fields are obtained by integrating (3) and (4) along the channel and its image.

\section{The coupling model for the calcu- lation of induced voltage}

For the coupling model, a set of time domain equations describing the voltages induced on multiconductors due to an electromagnetic field excitation as proposed by Agrawal[6] is used. According to this model, the forcing functions that excite the line are the horizontal and vertical components of the incident electric field.

For the conductors along the $x$-axis, the set of coupled differential equations describing the coupling between the electromagnetic field produced by the indirect lightning siroke and the multiconductors above the ground expressed in time domain are

$$
\begin{gathered}
\frac{\partial}{\partial x}\left[v_{i}^{s}(x, t)\right]+\left[R_{i j}\right]\left[i_{i}(x, t)\right]+\left[L_{i j}\right] \frac{\partial}{\partial t}\left[i_{i}(x, t)\right]=\left[E_{x}^{i}\left(x, h_{i}, t\right)\right] \\
\frac{\partial}{\partial x}\left[i_{i}(x, t)\right]+\left[G_{i j}\right]\left[v_{i}^{s}(x, t)\right]+\left[c_{i j}\right] \frac{\partial}{\partial t}\left[v_{i}^{s}(x, t)\right]=0
\end{gathered}
$$

where $\left[E_{x}^{i}\left(x, h_{i}, t\right)\right]$ is the vector of horizontal component of the incident electric field along the conductor at conductor height $h_{i}$. The sub index $i$ denotes the particular wire of the multiconductor line. $\left[R_{i j}\right],\left[L_{i j}\right],\left[G_{i j}\right]$ and $\left[C_{i j}\right]$ are the resistance, inductance, conductance and capacitance matrices per unit length of the line respectively. The sub index $i j$ denotes the mutual resistance, inductance, conductance and capacitance between $i$ th and $j$ th wires, respectively. $\left[i_{i}\right]$ is the line current vector. $\left[v_{i}^{s}\right]$ is the scattered voltage vector on the $i$ th conductor with respect to the ground. This is the voltage due to the field produced by the induced currents on the conductors. The scattered voltage is related to the total line voltage by the following relation.

$$
\left[v_{i}(x, t)\right]=\left[v_{i}^{s}(x, t)\right]+\left[v_{i}^{i}(x, t)\right]
$$

where $\left[v_{i}^{i}(x, t)\right]$ is the incident voltage.

$$
\left[v_{i}^{i}(x, t)\right]=-\int_{0}^{h_{i}} E_{z}^{i}(x, z, t) d z
$$

$E_{z}^{i}(x, z, t)$ is the incident(or inducing) vertical electric field at $x$ at a height of $z$.

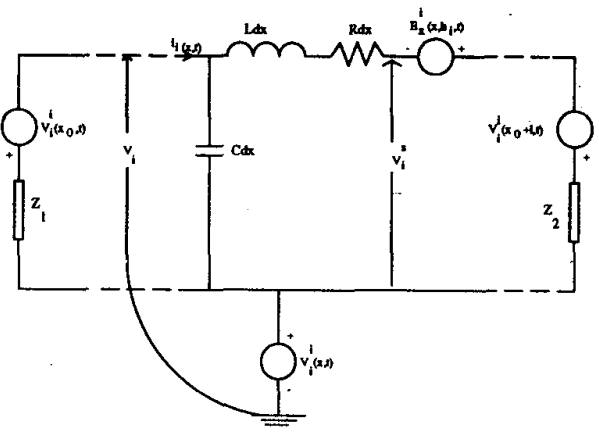

Figure 3: Equivalent circuit of a single-wire overhead line excited by lightning return-stroke electromagnetic field. Conductance is neglected.

The voltage at the end of the line is determined by the boundary condition and the current at the two ends of the line viz., $i\left(x_{0}, t\right)$ and $i\left(x_{0}+l, t\right)$, where $l$ is the span of the overhead line. The total voltage at both the line ends always have to be equal to $[i][z]$. Thus the boundary conditions for the scattered voltage are

$$
v_{i}^{s}\left(x_{0}, t\right)=-\left[Z_{1}\right]\left[i_{i}\left(x_{0}, t\right)\right]+\int_{0}^{h_{i}} E_{z}^{i}\left(x_{0}, z, t\right) d z
$$

$$
v_{i}^{s}\left(x_{0}+l, t\right)=\left[Z_{2}\right]\left[i_{i}\left(x_{0}+l, t\right)\right]+\int_{0}^{h_{i}} E_{z}^{i}\left(x_{0}+l, z, t\right) d z
$$

where $\left[Z_{1}\right]$ and $\left[Z_{2}\right]$ are the terminating impedence matrices.

For the calculation of the induced voltage on the transmission line due to lightning, if the height of the transmission line conductors $h_{i}$ are assumed to be small compared to the distance from the lightning strike then the vertical electric field can be considered as not varying in the height range $0<z<h_{i}$. Therefore $\left[v_{i}^{i}(x, t)\right]=-\int_{0}^{h_{i}} E_{z}^{i}(x, z, t) d z$ is approximated as $-\left[h_{i} \cdot E_{z}^{i}(x, 0, t)\right]$. But in this study the conductors are the connecting wires of the towers which are $120 \mathrm{~m}$ in height and for a lightning strike distance of $50 \mathrm{~m}, 200 \mathrm{~m}$ etc., the above approximation can not be applied and hence the inducing voltage $\left\{v_{i}^{i}(x, t)\right]$ is calculated by the integration $-\int_{0}^{h_{i}} E_{z}^{i}(x, z, t) d z$. The equivalent circuit for the case of a single wire above a perfect conducting ground excited by an incident electromagnetic field is shown in figure 3 . From the equation and the equivalent circuit we see that the forcing terms for the line currents and scattered voltages are the horizontal electric field at the location of the conductor in the absence of the conductors and the vertical electric field at line terminations only. To find the total voltage the vertical electric field at the point at which the line voltage is to be dertermined must be known.

The coupling equations (5) and (6) are solved by finite difference time domain(FDTD) technique. 
Proceedings of the International Conference on Electromagnetic Interference and Compatibility '99

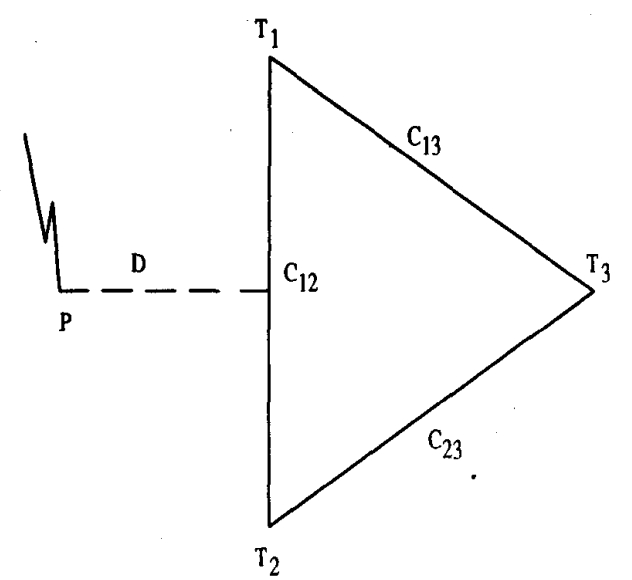

Figure 4: Sketch showing the protection system and the lightning striking point $(P)$, D- the striking distance.

\section{Calculation of induced voltage}

Induced voltage at the conductor mid span and at tower top due to lightning stroke at distances of $50 \mathrm{~m}, 200 \mathrm{~m}$ and $500 \mathrm{~m}$ are caiculated. Figure 4 shows the protection system and the lightning strike point. $T_{1}, T_{2}$ and $T_{3}$ are the lightning protection towers and $C_{12}, \mathrm{C}_{23}$ and $C_{13}$ are the conductors interlinking the towers.

The five bundled conductors connected between the towers are represented by an equvalent single conductor configuration. The equivalent circuit shown in figure 3 represents any one of the bundled conductor system, eg., the conductor $C_{12}$ connected between the towers $T_{1}$ and $T_{2}$, in which the voltage is to be calculated. The surge impedances of the towers $T_{1}$ and $T_{2}[7]$ are used as the terminating impedances $Z_{1}$ and $Z_{2}$ respectively. The conductors $C_{13}$ and $C_{23}$ are represented by their characteristic impedance terminated at $T_{1}$ and $T_{2}$. The induced voltages are calculated for the striking point distance $\mathrm{D}=50 \mathrm{~m}, 200 \mathrm{~m}$, and $500 \mathrm{~m}$ for a channel base current peak value of $i_{0}=10 \mathrm{kA}$. The results are shown in the figures 5,6 and 7 .

To see the effect of bundling the conductors, the induced voltage on yhe conductors are calculated for the peak current of $10 \mathrm{kA}$ and striking distance of $50 \mathrm{~m}$ with the conductors not bundled. Figure 8 shows the induced voltage waveform for the top and bottom conductors and at tower top.

Since the fields generated by the lightning stroke are distorted due to the finite conductivity of the ground, the induced voltage on the overhead conductors are also influenced by the conductivity of the ground. This distortion can be neglected when lightning strike distance is less. However for large lightning strike distances the ground conductivity influences significantly the horizontal component of electric field. To calculate the induced voltage including the ground conductivity, the horizontal electric field is calculated using Cooray-Rubinstein formula[8]. According to this formula the horizontal electric field with the finite ground conductivity $E_{r g}(z=h, r)$ is given

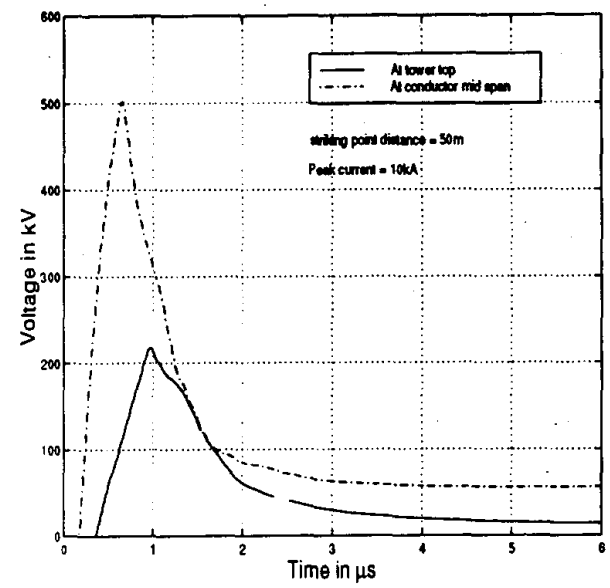

Figure 5: Induced voltage at tower top and at conductor midspan assuming ground as a perfect conductor, for a striking point distance of $50 \mathrm{~m}$.

as

$$
E_{r g}(z=h, r)=E_{r}(z=h, r)-H_{\phi}(z=0, r) \frac{\sqrt{\mu_{0}}}{\sqrt{\epsilon+\sigma / j \omega}}
$$

where $E_{r}(z=h, r)$ is the Fourier-Transform of the horizontal electric field at height $h$ and $H_{\phi}(z=0, r)$ is the FourierTransform of the azimuthal component of the magnetic field at ground level. Both $E_{r}(z=h, r)$ and $H_{\phi}(z=0, r)$ are calculated assuming a perfect conducting ground using equations (4), and (12) which is given as

$$
\begin{aligned}
& \mathrm{dH}_{\phi}(r, z, t)=\frac{d z^{\prime}}{4 \pi}[ \\
& \left.\frac{r}{c R^{2}} \frac{\partial}{\partial t} i\left(0, t-z^{\prime} / v-R / c\right)+\frac{r}{R^{3}} i\left(0, t-z^{\prime} / v-R / c\right)\right]
\end{aligned}
$$

where $\mu_{0}, \epsilon$ and $\sigma$ are the permeability of free space, permittivity and conductivity of the ground respectively.

Induced voltage has been calculated for a peak current of $i_{0}=200 \mathrm{kA}$ and for a striking distance $\mathrm{D}=500 \mathrm{~m}$ for (1) assuming the ground as perfect conductor and (2) with a finite ground conductivity $\sigma=0.001 \mathrm{~s} / \mathrm{m}$ and relative permittivity $\epsilon_{r}=10.0$. The voltage waveforms are shown in figures 9 and 10 respectively. A lightning return stroke peak current of $200 \mathrm{kA}$ has been chosen, as this is the highest recorded current to date and MIL STD $1757 \mathrm{~A}$ also stipulates this value for aero space systems. 
Kannu \& Thomas : Lightning Induced Voltages on Conductors Interlinking...

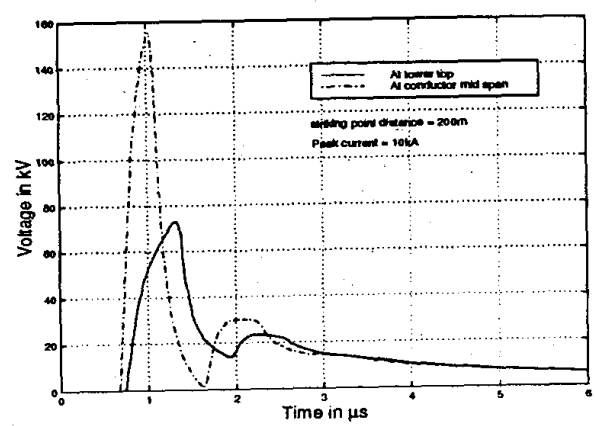

Figure 6: Induced voltage at tower top and at condur or midspan assuming ground as a perfect conductor, for a striking point distance of $200 \mathrm{~m}$.

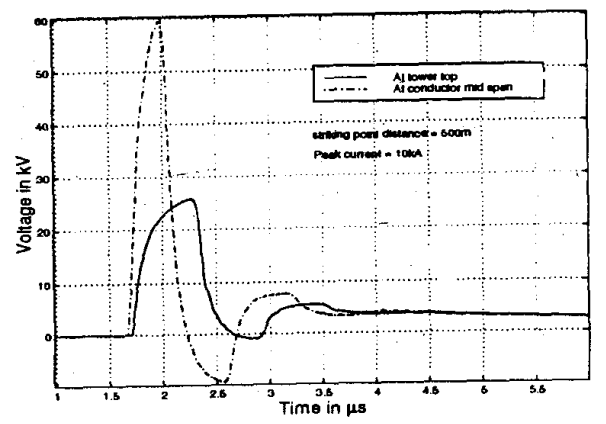

Figure 7: Induced voltage at tower top and at conductor midspan assuming ground as perfect conductor, for a striking point distance of $500 \mathrm{~m}$.

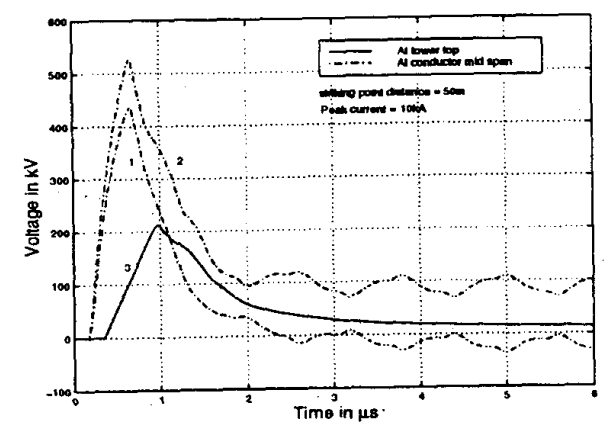

Figure 8: Induced voltage waveforms for the case where the conductors are not bundled and ground is considered as perfect conductor. 1- bottom conductor, 2- top conductor, and 3tower top.

\section{Results and discussion}

Figures 5, 6 and 7 show the induced voltage at mid-span of the conductor and at tower top for a lightning current of 10 $\mathrm{kA}$ and striking distances of 50,200 and $500 \mathrm{~m}$ respectively. The time delay seen in the waveforms corresponds to the time taken for the electromagnetic field produced by the lightning return stroke to travel to the respective observation points. The induced voltage for large striking point distance shows oscillations in the waveshape (eg., for $200 \mathrm{~m}$ and $500 \mathrm{~m}$ ). The oscillations are more for larger distances (compare figures 6 and 7). For the lightning striking distance of $500 \mathrm{~m}$ the oscillation even goes negative. This may be due to the fact that the difference in the time taken for the fields that arrive at the mid-span and tower top is less for large distances.

Figure 8 shows the induced voltage waveform for the case when the conductors are not bundled. The induced voltage at the conductor mid span is more on the top conductor where as for the bottom conductor it is less as compared to the bundled configuration. This is because the bottom conductors are shielded by the top conductors and hence the induced voltage is less. When the conductors are taken individually, the surge impedance becomes higher and hence a higher voltage is obtained as compared to a bundled conductor configuration. The induced voltage at the tower top is same for both the cases. It may be concluded that bundling the conductors in effect reduces the induced voltage.

The induced voltage waveform is calculated for a peak current of $i_{0}=200 \mathrm{kA}$ and distance $\mathrm{D}=500 \mathrm{~m}$ for (1) assuming the ground as perfect conductor and (2) with a finite ground conductivity of $0.001 \mathrm{~s} / \mathrm{m}$ and relative permittivity of 10.0 . The voltage waveforms are shown in figures 9 and 10 respectively. It is observed that the ground conductivity does not influence the magnitude and waveshape of the induced voltage. This is because, the conductor height is large and hence the distortion on the horizontal electric field due to finite conductivity is negligible.

\section{Conclusions}

The induced voltage at the tower top and mid-span of the connecting conductors between the towers of the lightning protection system due to a near by lightning return stroke has been calculated. Modified transmission line model for the lightning channel has been used to compute electromagnetic field. For computing the induced voltage the coupling model proposed by Agrawal et.al. has been used. The conductors are bundled and represented by single conductor configuration. It has been observed that the waveshape of the induced voltage depends on the lightning striking distance. Bundling the conductors reduces the magnitude of the induced voltage. The induced voltages are not influenced much by the earth conductivity.

\section{ACKNOWLEDGEMENT}

The authors would like to record their sincere thanks and appreciation to the authorities of Indian institute of science for the permission to publish this paper. 


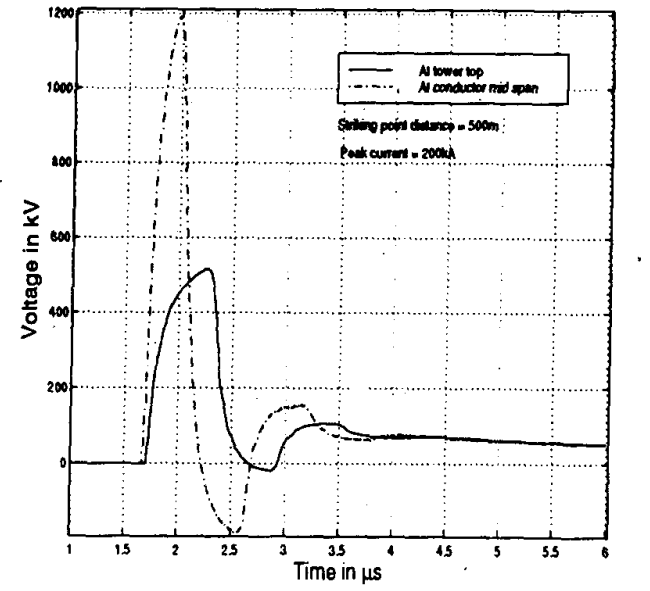

Figure 9: Induced voltage af, tower top and conductor midspan for a peak current of $200 \mathrm{kA}$ and a striking distance of $500 \mathrm{~m}$. Ground is assumed as a perfect conductor.

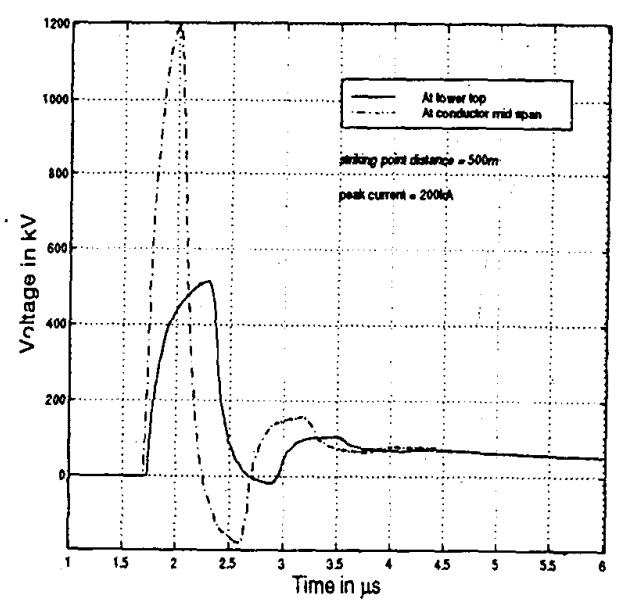

Figure 10: Induced voltage at tower top and conductor midspan for a peak current of $200 \mathrm{kA}$ and striking distance of $500 \mathrm{~m}$. Ground conductivity of $0.001 \mathrm{~s} / \mathrm{m}$ and relative permittivity of 10.0 have been assumed.

\section{References}

[1] Joy Thomas.M and G.R.Nagabhushana, "Development of a lightning protection scheme for satellite launch pad at Sriharikota", Report submitted to ISRO, 1997.

[2] C.A.Nucci,F,Rachidi,M,Ianoz and C.Mazzetti, "Lightning induced Voltages on Overhead Lines", IEEE Trans on EMC, Vol.35, No.1, pp 75-86, Feb 1993.

[3] A.Karwowski and A.Zeddam, "Transient Currents on Lightning Protection Systeme due to Indirect Lightning effect", IEE Proc-sci, Meas, Technol, Vol 142, No.3, pp 213-222, May 1995.

[4] M.J.Master and M.A.Uman, "Transient Electric and Magnetic Fields associated with establishing a finite Electrostatic Dipole", Am.J.I uys. 51, pp 118-126, Feb 1983.

[5] M.A.Uman, "Lightning Return Stroke Electric and Magnetic Fields", Jl .Geophy.Res, Vol.90, No D4, pp 61216130, June 1985.

[6] A.K.Agrawal, H.J.Price and S.J.Gurbaxani, "Transient Responce of Multiconductor Transmission Lines Excited by a Nonuniforr slectromagnetic Field", IEEE Trans on EMC, vol.22, No.2, pp 119-129, May 1980.

[7] Costa,F.S and Miranda,G,C, "Comparison of Lightning Tower Surge Models Using the EMTP', Tenth ISH, pp 327-330, August 1997.

[8] M.Rubinstein, "An approximate formula for the calculation of the horizontal electric field from lightning at close, intermediate and long ranges", IEEE Trans on EMC, vol.38, No.3, pp 531-535, August 1996. 